\title{
Incidental findings in a consecutive series of digital panoramic radiographs
}

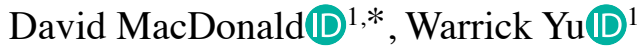 \\ ${ }^{1}$ Division of Oral and Maxillofacial Radiology, Faculty of Dentistry, University of British Columbia, Vancouver, British Columbia, Canada
}

\section{ABSTRACT}

\begin{abstract}
Purpose: The aim of this study was to determine the prevalence of incidental findings (IFs) on digital dental panoramic radiographs (DPRs) of asymptomatic patients attending a general dental practice.

Materials and Methods: This was a retrospective study of 6,252 consecutive digital (photostimulatable phosphor) DPRs of patients who visited a Canadian general dental practice for a complete new patient examination. The IFs were grouped into dental-related anomalies, radiopacities and radiopacities in the jaws, changes in the shape of the condyles, and other findings in the jaws, such as tonsilloliths and mucosal antral pseudocysts. Their prevalence was determined.

Results: Thirty-two percent of the DPRs showed at least 1 IF. The highest prevalence was found for dental-related anomalies (29\% of all DPRs), of which impacted teeth were the most prevalent finding (24\% of all DPRs), followed by idiopathic osteosclerosis (6\% of all DPRs). A lower prevalence was noted for tonsilloliths (3\%), and the prevalence of root tips, mucosal antral pseudocysts, and anomalies in condylar shape was approximately $1 \%$ each.

Conclusion: The observed prevalence of $32.1 \%$ for IFs of any type underscores the need for a dental practitioner to review the entire DPR when a patient presents for an initial dental examination (or check-up) or for dental hygiene. Only a single IF (a central giant cell granuloma) provoked alarm, as it was initially considered malignant. Similarly, impacted teeth and suspected cysts need careful evaluation upon discovery to determine how they may be optimally managed.(Imaging Sci Dent 2020; 50: 53-64)
\end{abstract}

KEY WORDS: Radiography, Panoramic; Jaw; Incidental Findings

\section{Introduction}

Although the focus of the general dental practitioner is upon the diagnosis and treatment of caries and periodontal disease and the subsequent restoration of the dentition, $\mathrm{s} / \mathrm{he}$ is responsible for detecting and identifying other disease processes such as inflammation and dysplastic, developmental, and neoplastic lesions. A recent radiological overview identified the most frequent and important lesions of the jaw bones that general dentists are likely to encounter during their professional lifetime. ${ }^{1}$ The central role the general dentist plays in the initial detection of these lesions cannot be over-emphasized. A recent example is of a case of non-Hodgkin lymphoma (NHL) in East Asia, in which

Received October 14, 2019; Revised February 7, 2020; Accepted February 10, 2020 *Correspondence to : Prof. David MacDonald

Division of Oral and Maxillofacial Radiology, Faculty of Dentistry, University of British Columbia, 2199 Wesbrook Mall, Vancouver V6 T 1 Z3, BC, Canada

Tel) 1-604-822-9762,E-mail) dmacdon@dentistry.ubc.ca a general dentist detected the abnormality and promptly began the diagnostic process, leading to its appropriate treatment. ${ }^{2}$ Malignancies arising within the alveolus may initially present as dental infections. ${ }^{3}$ Although the clinical examination is of paramount importance, it can only penetrate as far as the eye can see, the fingers can palpate, and dental instruments can probe, or to the extent that percussion and vitality testing can assess. To examine deeper, particularly into the bones of the jaws, requires radiography. Dental panoramic radiography (DPR) is a remarkably powerful tool that has become the mainstay imaging modality of most dental practices since the 1960s. The central role of DPR has not yet been displaced by the now ubiquitous availability of cone-beam computed tomography (CBCT) to the general dentist.

DPR is indicated after a clinical examination specifically to evaluate impacted teeth, oral lesions, such as cysts, tumours, or other pathological conditions of the jaws and jaw fractures. The major advantage of DPR is that both jaws 
can be displayed on a single image. Formerly, DPR had been used for general screening of new patients, but the universal application of the ALARA (as low as reasonably achievable) principle has emphasized the need to perform DPR only after a full clinical examination. Prior publications that identified the prevalence of incidental findings (IFs) observed on analog (film) DPR series were studies of American military entrants ${ }^{4}$ and new patients at a large British dental school. ${ }^{5}$ Both revealed large numbers of IFs, the most frequent of which were impacted teeth and radiolucencies.

More recently, film or analog technology has given way to digital technology, using either a charge-coupled device (CCD) sensor or a photostimulable phosphor (PSP) plate. The former does not use a cassette, whereas the latter still does. The latter technique has been used as a simple way to convert an analog DPR unit into a digital unit. These technologies have generally been associated with a reduction in the radiation dose and easier integration into patients' electronic medical records, and are also advantageous because they do not require chemical processing..$^{6-8}$ Additionally, exposure errors can be corrected digitally by adjusting the brightness. The latitude of PSP is greater and more linear than that of $\mathrm{CCD},{ }^{6-8}$ and its other advantages have recently been presented in the literature. ${ }^{6}$

The patients in this study came from a general dental practice in the Peace River Country region, which is located in the northwestern part of Alberta. This region covers an area of 110,854 square kilometres, and is larger in land mass than South Korea $\left(100,363 \mathrm{~km}^{2}\right)$. As of 2010 , it has an estimated population of 131,200 , which accounts for $3.5 \%$ of the total population of $3,600,000$ in the province of Alberta. ${ }^{9,10}$ The indigenous First Nations account for about $15 \%$ of the population, East Asians for $1.3 \%$, South Asians for $0.6 \%$ and Africans and Middle-Easterners for $0.9 \%$; the rest are of European origin.

Previous reports of IFs observed on CCD DPR have analyzed a selected dental hospital patient cohort of completely edentulous patients being considered for the provision of complete removable prothodontics. ${ }^{11,12}$ This study would appear to be first study of IFs observed on PDR using PSPs and also the first of patients attending a general dental practice solely for a dental examination or for dental hygiene.

The aim of this study was to determine, through a retrospective study the prevalence of IFs on PSP DPRs.

\section{Materials and Methods}

All digital radiographs for each patient were stored in an image folder on a file server. For the purposes of this study, only the DPRs associated with new patients who had mixed or permanent dentition and visited the office for a dental examination (also called a 'dental check-up') or for dental hygiene were considered. Therefore, patients presenting with any specific dental complaint were excluded.

The DPR unit was a Planmeca PM 2002 CC Proline device (Planmeca, Helsinki, Finland) (Fig. 2A). As image receptors, ScanX 5-inch $\times 12$-inch XO Pan (Air Techniques Inc., Melville, USA) PSP plates were used. After each exposure, the PSP was processed by a ScanX Classic processor (Air Techniques Inc.) (Fig. 2B), which converted the signal into an uncompressed digital image file in TIFF format. This TIFF file could then be read using the CaptureLink viewing software (version 10.11.11-Turbo; Henry Schein, Delta, BC, Canada).

All images were reviewed thoroughly by a single observer, using a Sharp (Model LL-127C-B, Mississauga, Canada) LCD monitor under reduced ambient lighting. The maximum luminance of this monitor was $300 \mathrm{~cd} / \mathrm{m}^{2}$ and the resolution was $1280 \times 1024$ pixels. During the viewing of each image, it was further enhanced by using the QuickHisto View function of the CaptureLink software to toggle the brightness and contrast of the image to ensure that all incidental radiolucencies and radiopacities were identified and were confirmed as not resulting from artefacts or processing errors. Certain clinically apparent findings such as implants, large caries, restorations, and impacted mandibular third molars that would have been visible clinically were not considered to be radiographic IFs. Other findings such as previously completed endodontic treatments and apicoectomy procedures that would have been part of the patient's known dental history were also not considered to be incidental.

The IFs detected on these radiographs were grouped into the following categories: dental-related anomalies (clinically undetected supernumerary teeth, congenitally missing teeth with retained primary teeth, impacted teeth, dilacerated roots, and residual root tips); radiopacities in the jaws (idiopathic osteosclerosis [IOS], condensing osteitis, osseous dysplasia, odontomas, and osteomas); abnormal findings around the condyles of the mandible (such as flattened condylar heads, osteophytes, bifid condyles, hypoplastic condyles and short condylar neck); and other findings that were clinically significant (tonsilloliths and mucosal antral pseudocysts).

Normal anatomical variants, such as tori mandibularis and calcified stylohyoid complex, were excluded.

The data in this study were categorical, and the Pearson 
chi-square $\left(\chi^{2}\right)$ test (with 1 degree of freedom) was used to determine the significance of differences according to sex within the same category of IF. It was determined that a difference in prevalence was statistically significant when $\chi^{2}>3.84(P<0.05 ; 2$-tailed $)$. Significant values are indicated in the tables with an asterisk $(*)$. Whenever the number of cases was inadequate for the $\chi^{2}$ test, "NA" was entered in the tables, meaning that the $\chi^{2}$ test could not be applied. The calculations were performed using VassarStats. ${ }^{13}$

Although ethics approval is not required for a retrospective study in Alberta, it was required by the University of British Columbia (Research Ethics Board approval number H12-02492), where the analysis was conducted.

\section{Results}

A consecutive series of 6,252 patients' images $(3,171$ males and 3,081 females) was identified. The age range of patients at the time of the exposures ranged from 7 to 90 years of age for males and from 7 to 88 years of age for females. Nearly one-third of patients were in the third decade of life (Table 1).

Of all the panoramic radiographs that were viewed for this study, 4,245 radiographs (67.9\%) had no IFs, and 2,007 radiographs $(32.1 \%)$ had 1 or more IFs. The findings and the corresponding prevalence rates are displayed in Table 2 .

The most frequent dental-related anomaly was a completely unerupted impacted tooth (Fig. 1A), which was found significantly more frequently in males. This predilection for males was largely due to the significantly greater prevalence of impacted mandibular third molars (Table 3).

Table 1. Distribution of male and female patients by age group

\begin{tabular}{rrrllr}
\hline Age group & Male & Female & $\chi^{2}$ & $P$ & Total \\
\hline $7-10$ & 132 & 150 & 1.81 & 0.178 & 282 \\
$11-20$ & 511 & 548 & 3.1 & 0.078 & 1,059 \\
$21-30$ & 989 & 936 & 0.45 & 0.488 & 1,925 \\
$31-40$ & 622 & 573 & 1.05 & 0.306 & 1,195 \\
$41-50$ & 503 & 476 & 0.2 & 0.655 & 979 \\
$51-60$ & 260 & 263 & 0.23 & 0.632 & 523 \\
$61-70$ & 113 & 73 & 7.72 & $0.005 *$ & 186 \\
$71-80$ & 26 & 44 & 5.22 & $0.022^{*}$ & 70 \\
$81-90$ & 15 & 18 & 0.37 & 0.543 & 33 \\
\hline Total & 3,171 & 3,081 & & & 6,252 \\
\hline
\end{tabular}

Table 2. Incidental findings and their predilection for male or female patients

\begin{tabular}{|c|c|c|c|c|c|c|}
\hline & Incidental findings & Male & Female & $\chi^{2}$ & $P$ & Total \\
\hline \multicolumn{2}{|c|}{ With at least 1 incidental finding } & $1,122(17.9 \%)$ & $885(14.2 \%)$ & 31.8 & $<0.05$ & $2,007(32.1 \%)$ \\
\hline \multicolumn{2}{|c|}{ Dental-related anomalies } & $987(15.8 \%)$ & $832(13.3 \%)$ & 12.9 & $<0.05$ & $1,819(29.1 \%)$ \\
\hline \multirow{7}{*}{$\begin{array}{l}\text { Dental-related } \\
\text { anomalies }\end{array}$} & Impacted tooth & $797(12.7 \%)$ & $673(10.8 \%)$ & 9.4 & $<0.05$ & $1,470(23.5 \%)$ \\
\hline & $\begin{array}{l}\text { Superimposition of third molars on } \\
\text { mandibular canal }\end{array}$ & $113(1.8 \%)$ & $95(1.5 \%)$ & 1.1 & 0.069 & $208(3.3 \%)$ \\
\hline & Supernumerary tooth & $30(0.3 \%)$ & $23(0.3 \%)$ & 1.4 & 0.390 & $51(0.8 \%)$ \\
\hline & Congenitally missing permanent tooth & $48(0.8 \%)$ & $46(0.7 \%)$ & $<0.0$ & 1.000 & $94(1.5 \%)$ \\
\hline & Dilacerated root & $27(0.4 \%)$ & $25(0.4 \%)$ & $<0.0$ & 0.862 & $52(0.8 \%)$ \\
\hline & Root tips embedded in alveolar bone & $52(0.8 \%)$ & $33(0.5 \%)$ & 3.8 & $<0.05$ & $85(1.3 \%)$ \\
\hline & Root resorption & $8(0.32 \%)$ & $3(0.12 \%)$ & NA & NA & $11(0.2 \%)$ \\
\hline \multirow{2}{*}{$\begin{array}{l}\text { Alveolar } \\
\text { radiopacities }\end{array}$} & Idiopathic osteosclerosis & $196(3.1 \%)$ & $180(2.9 \%)$ & 0.3 & 0.572 & $376(6.0 \%)$ \\
\hline & Sclerosing osteitis & $35(0.6 \%)$ & $30(0.4 \%)$ & 0.3 & 0.610 & $65(1.0 \%)$ \\
\hline \multirow{3}{*}{$\begin{array}{l}\text { Radiolucent areas } \\
\text { in the jaw bones }\end{array}$} & Possible periapical or residual cyst & $16(0.3 \%)$ & $11(0.1 \%)$ & 0.8 & 0.374 & $27(0.4 \%)$ \\
\hline & Possible dentigerous cyst & $11(0.2 \%)$ & $7(0.1 \%)$ & 0.8 & 0.377 & $18(0.3 \%)$ \\
\hline & Lingual bone defect & $2(0.0 \%)$ & $1(0.0 \%)$ & NA & NA & $3(0.0 \%)$ \\
\hline \multirow[t]{5}{*}{ Other anomalies } & Tonsillolith & $103(3.2 \%)$ & $78(2.5 \%)$ & 2.9 & 0.091 & $181(2.9 \%)$ \\
\hline & Sialolith & $2(0.1 \%)$ & $3(0.1 \%)$ & NA & NA & $5(0.1 \%)$ \\
\hline & Metal fixation & $42(0.7 \%)$ & $25(0.4 \%)$ & 3.9 & $<0.05$ & $67(1.1 \%)$ \\
\hline & Mucosal antral pseudocyst & $32(0.5 \%)$ & $40(0.6 \%)$ & 1.2 & 0.284 & $72(1.1 \%)$ \\
\hline & Anomalies in the shape of the condyle & $29(0.5 \%)$ & $28(0.4 \%)$ & $<0.0$ & 0.920 & $57(0.9 \%)$ \\
\hline
\end{tabular}



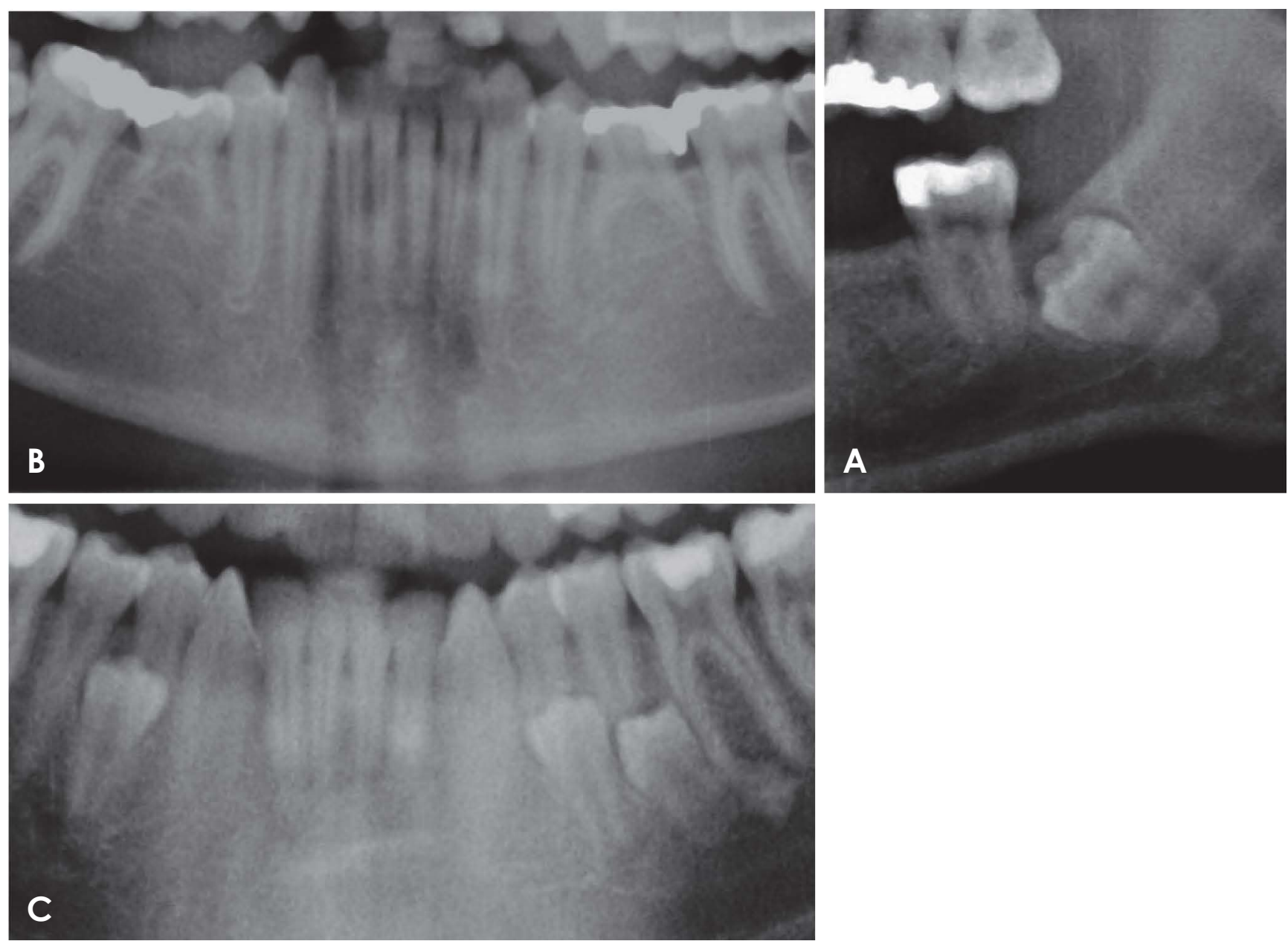

Fig. 1. Dental-related anomalies. A. An impacted left mandibular third molar, intimately related to the mandibular canal, displacing it downward and reducing its diameter. B. Congenitally missing premolars bilaterally. C. Supernumery mandibular premolars. The left first molar has a radiopacity at its mesial root, which in conjunction with the moderately large restoration should prompt a vitality test in order to distinguish between idiopathic osteosclerosis, which needs no treatment, and condensing osteitis, which does.

Some lower third molars were found to have intimate relationships with the corresponding inferior dental canals (Table 2). There was a high degree of variation in the relationship between the third molar and the inferior dental canal. Just over $3 \%$ of lower third molars showed either a radiolucent band across the roots (Fig. 1A) or loss of the cortical border of the inferior dental canal in the regions where the roots were superposed.

The second most common dental-related anomaly was found to be congenitally missing permanent teeth and retained primary dentition (Fig. 1B). Due to high frequency of tooth extractions in the Peace River Country region in all age groups, for the purpose of this study, in order to positively identify a congenitally missing tooth, the retained primary counterparts must have been present in the adult dentition. Most of these congenitally missing permanent teeth were lower second premolars with the deciduous second molars as their retained counterparts, and there were 80 such cases (Fig. 1B).

The lower first and second premolars were equally common locations of supernumerary teeth (Fig. 1C). Supernumerary teeth were also found in other regions.

Unusual tooth anatomy could also be found on DPRs as dental-related anomalies, such as dilacerated roots (Fig. 2A). In this study, there were 85 DPRs (1.4\%) showing 1 or more residual root tips that were embedded in the alveolar bone (Fig. 2B) and there was a predilection for males. These root tips most likely occurred as a result of incomplete extractions. Root resorption (Fig. 2C) was observed in only 11 cases.

The most common type of radiopacity was the IOS, which is a deposition of bone of unknown aetiology around 

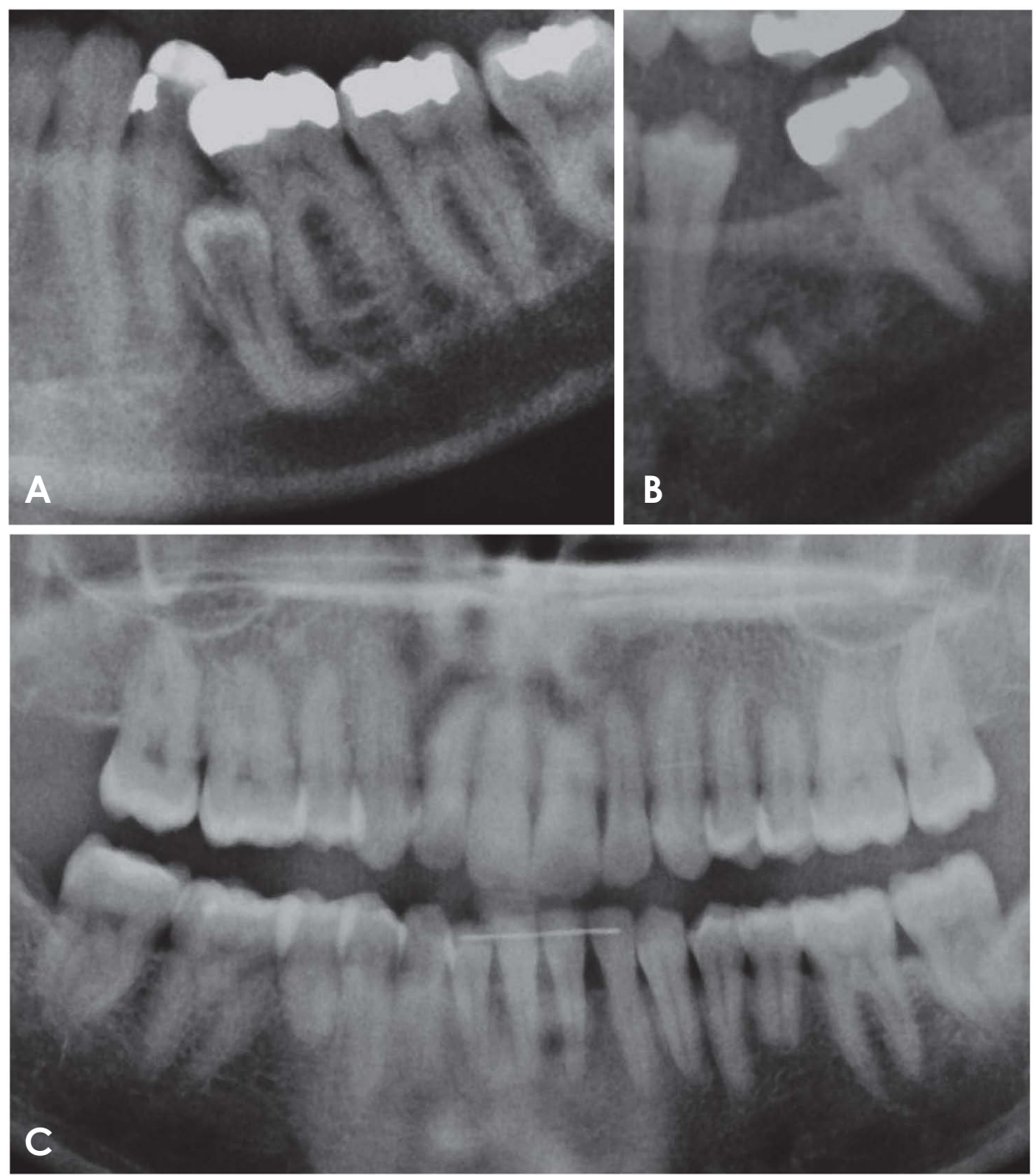

Fig. 2. Dental-related anomalies. A. An impacted right second premolar with a right-angled dilaceration of its root. B. A deeply-buried root apex from an extracted first molar. The radiolucency substantially enclosing it is suggestive of an ongoing infection. The premolar root displays a slight dilaceration, less severe than that in A. C. A post-orthodontically treated patient displaying root resorption of the premolars. A retainer wire has been cemented to the lower incisors. A notable finding is the small radiopacity between the roots of the maxillary first molar and the presumably second premolar. This is an idiopathic osteosclerosis, which are infrequently observed in the maxilla.

the trabeculae (Fig. 3A). ${ }^{14,15}$ This condition is also known as enostosis or dense bony island. ${ }^{14,15}$ In this study, radiopacities in the jaw were diagnosed as IOS according to MacDonald-Jankowski's criteria. ${ }^{15}$ In this study, all cases of IOS but 1 were detected in the alveolar bone of the mandible. Figure $2 \mathrm{C}$ displays the sole IOS in the maxillary alveolus. Table 4 shows the distribution of IOS in the mandible. The most common location of IOS was in the molar regions of the mandible, followed by the premolar regions and then by the parasymphyseal regions (Table 4). In all cases, IOS was only found in the alveolus, and there was no sex predilection.

Radiopaque lesions associated with carious or deeply restored tooth are more likely to be sclerosing osteitis (also known as condensing osteitis) (Fig. 3B). The histopathological features of sclerosing osteitis are the same as those of IOS. ${ }^{14}$ The radiological appearance of sclerosing osteitis appears the same; however, it is of low-grade inflammatory origin $^{14,15}$ and the condition may require treatment. ${ }^{15}$

Periapical radiolucencies of inflammatory origin (PRIOs) are unilocular radiolucencies associated with the apices of non-vital teeth. Most are small (Fig. 4A) and on occasion may be surrounded by a band of condensing osteitis (Fig. 4A). While they can only be determined definitively to be either periapical granulomas or periapical (or radicular) cysts histologically, PRIOs measuring $1.5 \mathrm{~cm}$ or more in diameter on radiography are considered to be periapical cysts. ${ }^{6}$ Figure 4B indicates such a lesion, which was associated with the apices of an endodontically-treated molar and was so large that it displaced the second molar. According to this criterion, there were 24 cases of possible periapical cysts (Fig. 4B). Dentigerous cysts are the second most frequent type of cysts. These are pericoronal unilocular radiolucencies arising from the cementoenamel junction. 

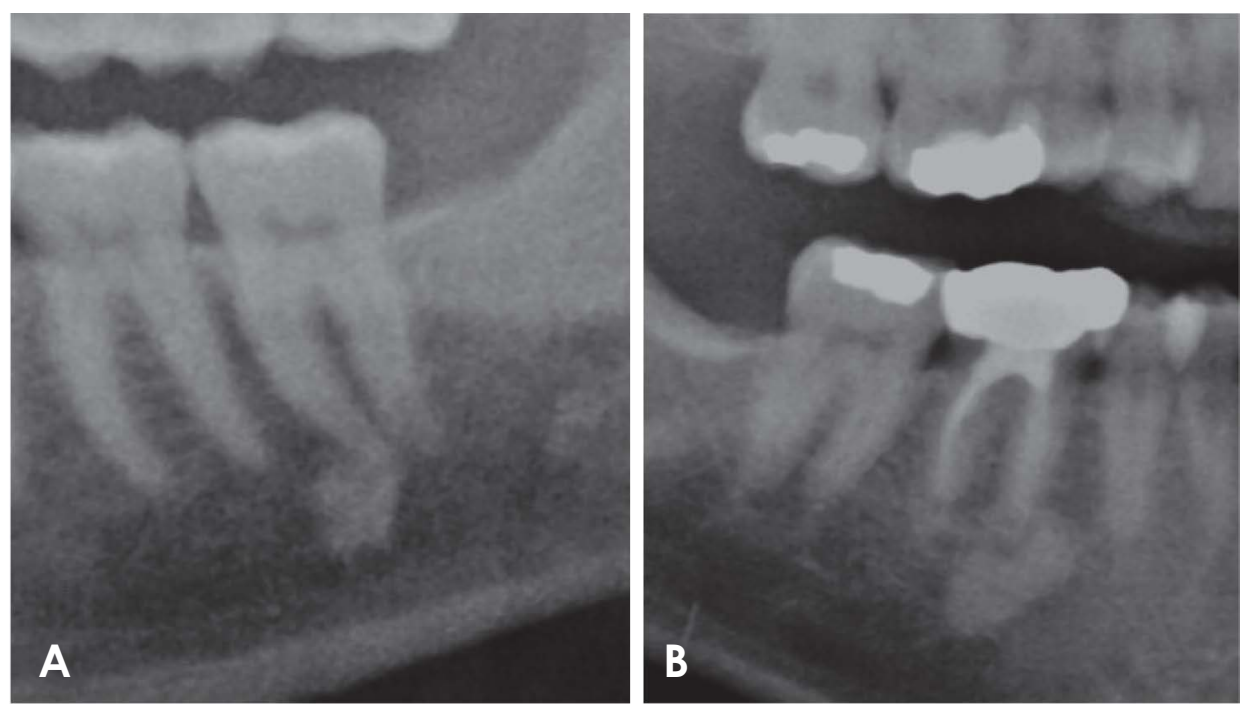

Fig. 3. Radiopacities. A. Idiopathic osteosclerosis associated with the apex of a molar tooth that displays no caries or large restorations that may suggest pulpal necrosis. B. Sclerosing osteitis associated with a periapical radiolucency at the apex of a root-filled molar tooth.

Table 3. Distribution of impacted teeth within the jaws of male and female patients

\begin{tabular}{|c|c|c|c|c|c|}
\hline Impacted teeth & Male & Female & $\chi^{2}$ & $P$ & Total \\
\hline Lower third molars & 577 & 484 & 6.86 & $<0.05$ & $1,061(72.2 \%)$ \\
\hline Upper third molars & 173 & 159 & 1.25 & 0.264 & $332(22.5 \%)$ \\
\hline Upper canines & 31 & 19 & 3.35 & 0.067 & $50(3.4 \%)$ \\
\hline Lower canines & 6 & 4 & NA & NA & $10(0.7 \%)$ \\
\hline Upper second molars & 1 & 0 & NA & NA & $1(<0.1 \%)$ \\
\hline Lower second molars & 3 & 0 & NA & NA & $3(0.2 \%)$ \\
\hline Lower first molars & 0 & 1 & NA & NA & $1(<0.1 \%)$ \\
\hline Upper second premolars & 2 & 3 & NA & NA & $5(0.3 \%)$ \\
\hline Lower second premolars & 2 & 3 & NA & NA & $5(0.3 \%)$ \\
\hline Upper incisors & 2 & 0 & NA & NA & $2(0.1 \%)$ \\
\hline Total & 797 & 673 & & & $1,470(100.0 \%)$ \\
\hline
\end{tabular}

Table 4. Distribution of idiopathic osteosclerosis within the alveolus of the mandible

\begin{tabular}{crrrrr}
\hline Distribution of location for idiopathic osteosclerosis & Male & Female & $\chi^{2}$ & $P$ & Total \\
\hline Mandibular molar region & 148 & 125 & 1.4 & 0.238 \\
Mandibular premolar region & 44 & 52 & 0.9 & 0.335 \\
Mandibular parasymphyseal region & 4 & 3 & NA & NA \\
\hline Total & 197 & 180 & 0.3 & 0.572 \\
\hline
\end{tabular}

A case of a possible dentigerous cyst is displayed in Figure 4C. The other radiolucencies displayed in Figure 4D were a case of lingual bone defect (LBD, earlier known as Stafne's bone cysts) (Fig. 4D) and a central giant cell granuloma (CGCG) (Fig. 5). The CGCG was confirmed by histopathology.

Tonsilloliths result from dystrophic calcification in the tonsils. ${ }^{16}$ Although there are 3 groups of tonsils (namely, the palatine, pharyngeal and lingual tonsils), the palatine tonsils are the most conspicuous on DPR, where they are superposed on the vertical ramus of the mandible.

Sialoliths can have varying amounts of organic and inorganic materials. ${ }^{17}$ When these stones are sufficiently calcified, they can become radiopaque and appear on DPR. 

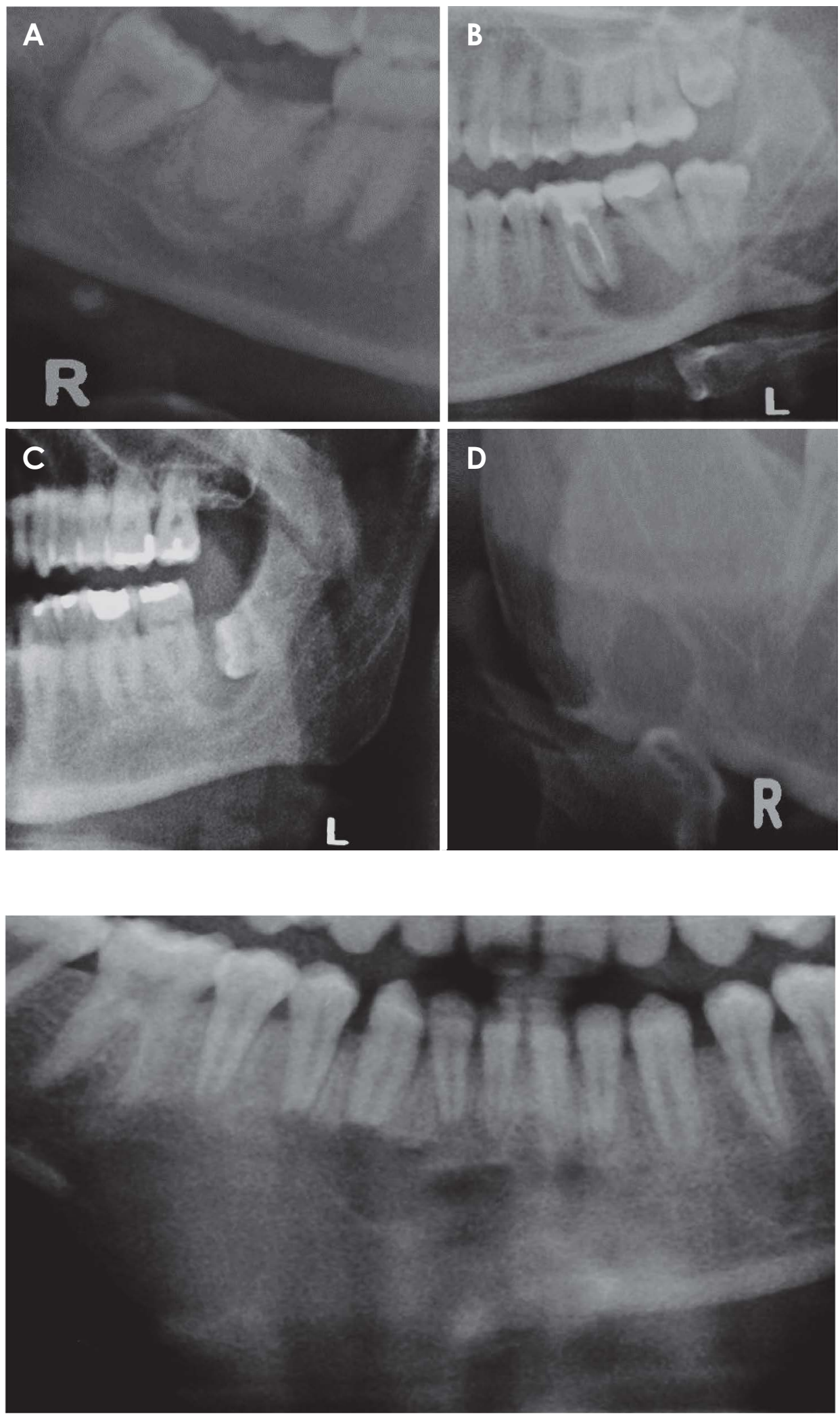

Fig. 4. Radiolucent areas in the bones of the jaws. A. A periapical radiolucency at the first right decayed molar. It is surrounded by a band of condensing osteosclerosis which has extended slightly into the basal process, accentuating the mandibular canal. B. A periapical radiolucent of inflammatory origin associated with the root-filled first molar. It has displaced the mandibular canal downwards and the root of the second molar backwards. C. A pericoronal radiolucency on a unerupted third molar is consistent with a dentigerous cyst. D. A lingual bone defect sited between the lower border of the mandible and mandibular canal.

Fig. 5. A central giant cell granuloma extending from the midline of the mandible to the right first molar. It has resorbed the apices of the right premolars and anterior teeth and has expanded and eroded the lower border of the mandible.
Only those that appeared within the submandibular soft tissue (i.e., below the lower border of the mandible on DPR) (Fig. 4A), could be reasonably determined to be sialoliths in this study.

Mucosal antral pseudocysts were the most common finding in the maxillary sinuses. These are well-defined dome- 

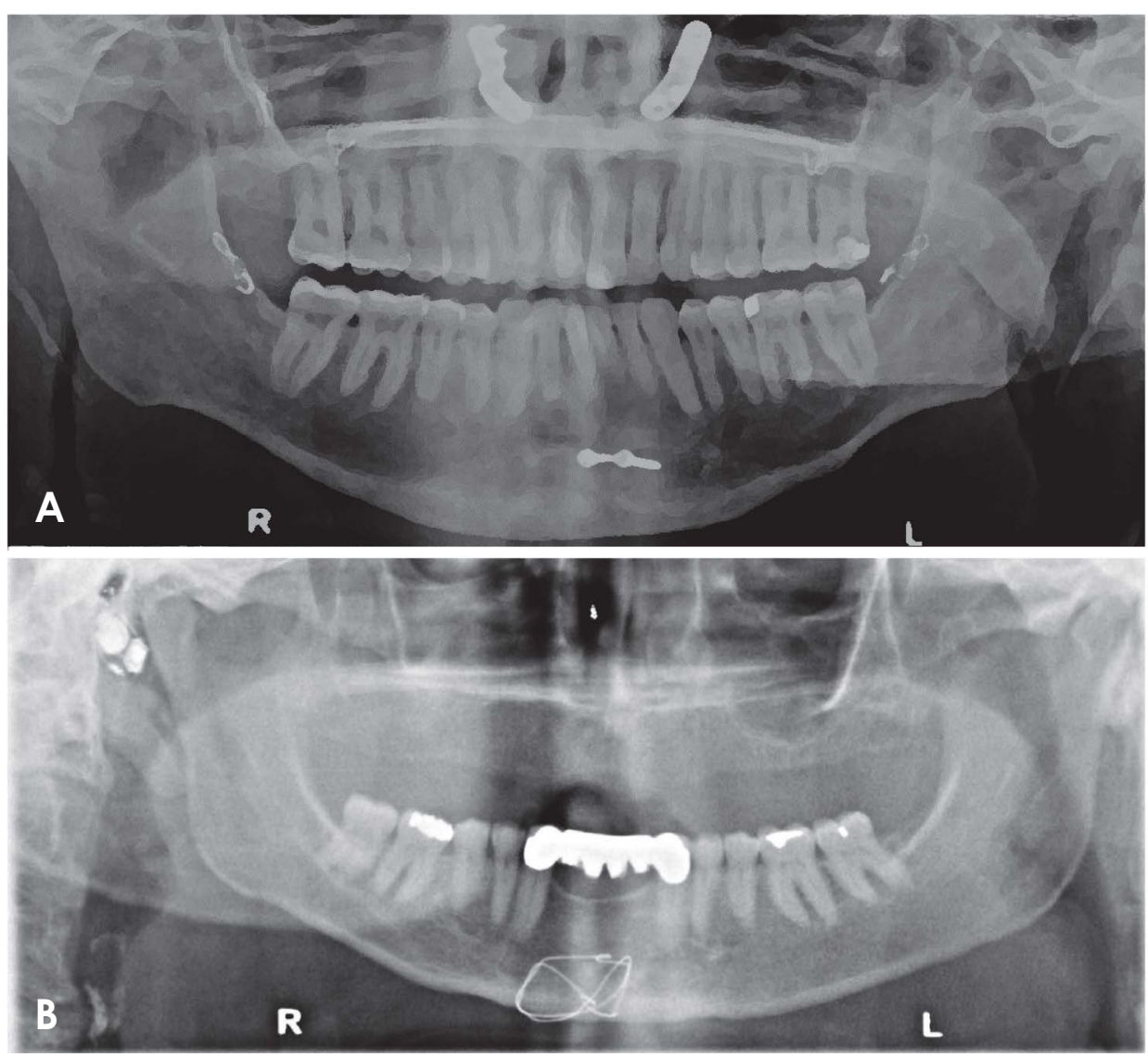

Fig. 6. Metal fixtures and non dental implants. A. Fixation bimaxillary osteotomy; Le Fort 1, Obwegeser type osteotomy and genioplasty. B. Wire fixation of the chin. Of note, a hearing aid is present in the right ear and a right calcified carotid artery atheroma is located anterior to the vertebral bodies. shaped opacities arising from the floor of the maxillary sinus. ${ }^{6,18,19}$

Although the temporomandibular joint (TMJ) is seen on DPR, only anomalies of the condyles can be displayed, whereas the articular eminence and fossa cannot be evaluated adequately due to superposition of the base of the skull and the zygomatic arch. These anomalies were flattened (27 cases), osteophytic (11 cases), bifid (14 cases), and hypoplastic (4 cases) condyles. ${ }^{20,21}$

Although it is reasonable to expect a patient to report a previous fracture or operation, disclosure of the degree of retention of the fixation from these events is not predictable, and the display of the positioning and extent of that fixation on DPR can constitute, in effect, an IF that may change the treatment plan. There were 67 DPRs (1.1\%) with single or multiple metal fixtures in the mandible, maxilla, or both (Fig. 6). These fixtures included metal plates or wires to fix previously fractured bone prior to the subjects' initial visit to this clinic. There was a significantly higher prevalence of these fixtures in males $\left(\chi^{2}=3.9\right)$.

Suspected cysts were referred to oral and maxillofacial surgeons for further investigation and treatment. The only clinically significant lesion was the CGCG (Fig. 5), which affected a teenager and was initially thought to be a malignancy.

\section{Discussion}

Digital DPR has many advantages over film-based panoramic radiography. ${ }^{6}$ However, a high-quality digital radiograph alone is not adequate to make an accurate diagnosis, as the viewing conditions of the radiograph are just as important. For film-based analog radiography, the viewing conditions include a bright light-box with a luminance (brightness) of $1500-3000 \mathrm{~cd} / \mathrm{m}^{2}$, as well as an illuminance (reduced ambient lighting) of 100 lux or less. In digital radiography, the display monitor takes the role of the lightbox used for analog radiography. The American College of Radiology recommended a luminance of $171.3 \mathrm{~cd} / \mathrm{m}^{2}$ for viewing digital radiographs. ${ }^{6,8}$ The computer monitor used in this study was set to a maximum luminance of $300 \mathrm{~cd} /$ $\mathrm{m}^{2}$. Although this was higher than most monitors used in general practice at the time of the study, the viewing conditions in this study were generally comparable to those of private Canadian dental clinics with digital systems, as most general dentists have little or no advanced training 
in oral and maxillofacial radiology and therefore in reality would not be aware of the viewing conditions of digital radiographs used by radiologists.

Three types of IFs - namely, unerupted impacted teeth (Fig. 1A), retained root tips (Fig. 2B), and metal fixations (Fig. 6) - showed a significantly higher prevalence in males than females. The higher prevalence of unerupted impacted teeth in males could have been due to the larger number of male subjects in this study. ${ }^{22}$ There were also significantly more female patients with a history of orthodontic treatment, ${ }^{22}$ which also influenced the prevalence of impacted teeth. As a result of these factors, female subjects were more likely to have already had their impacted teeth removed or to have had more space orthodontically created for them to erupt into prior to becoming new patients at this clinic; therefore, a lower prevalence of unerupted impacted teeth was observed in female subjects.

Impacted teeth were the most prevalent dental-related anomaly. A tooth is considered to be impacted when it has not fully erupted to the normal functional position in the occlusal plane. For the purposes of this study, in order to avoid including impacted teeth that may appear impacted on a panoramic radiograph but are actually clinically visible, the definition of a radiological IF of an impacted tooth was a mature tooth with full bony coverage. In order for the unerupted tooth to be definitively diagnosed as an impacted tooth, in addition to the complete formation of its roots, alveolar bone must be present occlusal to it.

Third molars in this study were significantly less frequently observed than in a Chinese study $\left(82.5 \%, \chi^{2}=72.7\right.$ for lower third molars). ${ }^{23}$ A possible reason for the generally low prevalence is that many partially erupted impacted teeth were rigorously excluded from the count, as they would have been detected clinically and were not considered radiological IFs. It is also reasonable to surmise that many patients' unerupted third molars had been detected by a previous dentist and removed prior to their initial visit to the present practice. Support for this comes from Table 1 in which the overwhelming majority of new patients first presented at this practice during their third decade of life or at an older age.

The low number of retained root tips may reflect the decreasing level of dental decay in Canadians over recent decades. ${ }^{24}$ Regarding the higher prevalence of fixtures in males than in females, it can be speculated that males were more likely to acquire plates and wires to repair fractures sustained in the course of altercations (i.e., fights) or at work, as the Peace River Country region has the highest rate of hospital admissions for injuries in Alberta. ${ }^{25}$
IOS accounted for $78 \%$ of all radiopacities. A systematic review reported that the prevalence of IOS ranged from $2.7 \%$ to $6.7 \%$, with higher prevalence in Chinese from Hong Kong. ${ }^{15}$ Using the same criteria as that systematic review, this study found that $6.0 \%$ of all the DPRs displayed IOS, a number that falls within the previously reported range, although the population in the Peace River Country region is primarily of European origin. One explanation is fluoridation of the water supply. Of note, in Hong Kong, the concentration of fluoride in the water changed from $1.0 \mathrm{ppm}$ to $0.7 \mathrm{ppm}$ during the 10 -year period of that study, which was accompanied by a significant decrease in both the prevalence and size of IOS. ${ }^{15}$ Thus, higher levels of fluoride may contribute to a higher prevalence of IOS, and it is well known that the water supply in the Peace River Country region (as well as Alberta in general) contains 0.5 to $1.5 \mathrm{ppm}$ of naturally occurring fluoride content. ${ }^{26}$

An important feature of IOS is that it occurs more frequently in the premolar region. This feature was reported in the aforementioned systematic review. ${ }^{15}$ As an explanation of this phenomenon, it has been suggested that sclerotic bone forms as a result of previous retained deciduous molar roots that are resorbed and replaced by sclerotic bone. ${ }^{15}$ This may explain the IOS discovered in Figure 2C. As the majority of cases of IOS in this study were found in the molar region, an as-of-yet undetermined cause may be responsible for the distribution of this feature in this Canadian community.

Osseous dysplasia is most frequently found in middleto old-age women of East Asian and sub-Saharan African origin. ${ }^{6}$ No cases of osseous dysplasia were observed. This is not surprising since the proportion of individuals of East Asian and sub-Saharan African origin was very low in this population, the overwhelming majority $(70 \%)$ were younger than 40 years of age, and over half were male.

In a recent study, the identity and the relative period prevalence of the most frequent lesions biopsied in general dental practice were tabulated. ${ }^{27}$ All of them would have presented as radiolucencies. ${ }^{27}$ Radiolucencies, particularly PRIOs, constitute the greatest focus of the general dentist with regard to lesions within the jaws, as they indicate dental problems that are the frequent source of dental complaints. The ability to address these complaints is within the expertise of the general dentist. Their low prevalence in this study reflects the fact that patients presenting with a dental complaint were excluded from this study. Radiolucencies that appear to be dentigerous cysts are generally referred to an oral and maxillofacial surgeon, particularly when they may present difficulties (e.g., if they are located 
deep within the jaws).

LBD is a radiolucency that poses a potential risk of mandible fracture. ${ }^{1}$ Only 3 such cases were identified in this study $(0.05 \%$; 1 out every of 2000 DPRs), which is lower than was reported in any of the 10 reports synthesized by Assaf et al. ${ }^{28}$ The prevalence reported in those studies ranged from $0.08 \%$ ( 1 out of every 1200 DPRs) ${ }^{29}$ to $0.48 \%$ (1 out of every 200 DPRs). ${ }^{30}$ The latter rate was reported in a case series that entirely comprised men aged 40 years and over. ${ }^{30}$ Sisman et al. reported that only $14 \%$ of cases of LBDs were in females (their overall prevalence was $0.08 \%)^{29}{ }^{29}$ Therefore the lower prevalence of LBDs in this study may reflect both the fact that $70 \%$ of the subjects were younger than 40 years of age and almost half were female.

Tonsilloliths in the palatine tonsil appear on DPR as radiopacities superimposed upon the vertical ramus. Takahashi et al. reported a prevalence of $13.40 \%$ in a Japanese sample, ${ }^{16}$ whereas Aoun et al. reported a prevalence of $7.25 \%$ in a Lebanese study. ${ }^{31}$ Aoun et al. stated that the diagnosis of tonsilloliths is important in cases of non-specific chronic halitosis. 31

This study yielded 5 cases of sialoliths, and all showed a radiopaque mass in the submandibular space inferior to the body of the mandible, indicating a sialolith in the submandibular glands. Although this rate is less than the prevalence of $1.2 \%$ discovered as IFs on autopsy, ${ }^{32}$ any sialoliths present elsewhere in the submandibular duct system may have been obscured by the body of the mandible or mistaken as an IOS or sclerosis osteitis. Despite the latter possibility, both IOS and sclerosing osteitis were found in the alveolus and not in the basal process. Although it was very infrequent for a sialolith of considerable size to be detected on DPR in an asymptomatic patient (as symptomatic patients were excluded from this study), ultrasonography detected submandibular sialoliths as IFs in $6 \%$ of cases. ${ }^{32}$ The concern has been raised that sialoliths may be linked to lithiasis elsewhere in the body. Reassuringly, a recent Korean study reported that no association was found between cholelithiasis (gallstones) and sialolithiasis. ${ }^{33}$

Mucosal antral pseudocysts were found in $1.1 \%$ of all DPRs, which is lower than the prevalence of $5 \%$ to $14 \%$ reported in studies in other parts of the world. ${ }^{18,19,34} \mathrm{Mu}-$ cosal antral pseudocysts are known to be related to cold air, pollutants in the air, and central heating. ${ }^{34}$ A significant proportion of workers in the Peace River Country work outdoors year-round. Therefore, while the primary predisposing factor for mucosal antral pseudocysts in this region is cold air, this would appear to have been offset by not liv- ing in a polluted urban environment.

A major departure from other studies, that reported a male predilection of mucosal antral pseudocysts, in the present study more were found in females. Furthermore, a possible source of error leading to a low reported prevalence in this study may be the inclusion of 759 subjects (12.1\% of all subjects) who were 7 to 14 years of age, whereas other studies analyzed only individuals older than $15^{18,19}$ or 30 years of age. ${ }^{34}$ The maxillary sinuses are not fully developed until the age of $12,{ }^{6}$ and mucosal antral pseudocysts are therefore unlikely in subjects under the age of 14 , contributing to their low reported prevalence in this study. They occur most frequently in the third decade of life. $^{18,19}$

Changes in condylar shape may reflect a past history of temporomandibular disease in patients who were complaint-free - and presumably also symptom-free - at the time of DPR. These changes on the shape of the condylar head are not the only potentially misleading imaging features within the TMJ. Joint effusion in the TMJ was once considered a reliable indicator of inflammation in the TMJ when observed on T2-weighted magnetic resonance imaging, but it is now only considered a sign of pathology if the amount of fluid exceeds that of asymptomatic individuals. ${ }^{35}$

Figure 6B displays a calcified carotid artery atheroma (CCAA) in a patient who already had a significant cardiac history ( 3 coronary bypasses); therefore, this CCAA would not be an IF in the true sense. It exhibited a distinctive curvilinear radiopaque appearance. ${ }^{6,36}$ Nevertheless, other entities in the same region of the neck may mimic CCAA on DPR, yielding false positives. ${ }^{6,36}$ Therefore, although DPR can be useful in detecting CCAA, its high false positive and false negative rates mean that DPR should not be considered to be a reliable method of detecting CCAA. ${ }^{6,36}$ Nevertheless, a dentist must be knowledgeable regarding CCAA as an IF on DPR so that appropriate management steps can be taken. The detection of a sustained elevated blood pressure during routine blood pressure measurements of a new or check-up patient, which is part of the standard of care in most North American jurisdictions, may indicate that the patient might be at risk for a cerebrovascular accident (also known as stroke). ${ }^{6,36}$ Under both circumstances, a referral to the patient's medical doctor is necessary for further investigation, as discussed elsewhere. ${ }^{6,36}$

In conclusion, this study is the largest reported consecutive series of digital DPRs from patients who did not present with a dental complaint. Nevertheless, $0.5 \%$ and $1.0 \%$ of the DPRs revealed periapical radiolucencies of inflammatory origin and condensing osteitis, respectively. These 
findings indicate non-vital teeth in need of treatment.

Almost a quarter of DPRs had impacted teeth, and 3\% of the DPRs exhibited mandibular third molars superimposed on the mandibular canals (Fig. 1A). This feature may be an indication for CBCT.

The prevalence of IOS was similar to that in Hong Kong, but higher than that in London. ${ }^{15}$

The prevalence of mucosal antral pseudocysts in Alberta was markedly lower than that in other reports. ${ }^{18,19}$

The ability to optimize the display of each digital DPR may contribute to some of the differences observed in comparisons with studies that analyzed analog DPRs.

Although almost one-third of the DPRs displayed at least $1 \mathrm{IF}$, the overwhelming majority were not suggestive of an oral lesion with a potentially serious outcome. Nevertheless, such lesions do occur, as exemplified by the case of CGCG(Fig. 5).

\section{Conflicts of Interest: None}

\section{Acknowledgements}

This study was performed for a partial fulfilment of a MSc in Dental Radiology at the University of London. The authors are very grateful to Drs. Ng and Ngu of King's College London, United Kingdom and to the Northern Dental \& Hygiene Centre, Grande Prairie, Alberta, Canada.

\section{References}

1. MacDonald D. The most frequent and/or important lesions that affect the face and the jaws. Oral Radiol 2020; 36: 1-17.

2. MacDonald D, Li T, Leung SF, Curtin J, Yeung A, Martin MA. Extranodal lymphoma arising within the maxillary alveolus: a case report. Oral Surg Oral Med Oral Pathol Oral Radiol 2017; 124: e233-8.

3. MacDonald D, Lim S. Extranodal lymphoma arising within the maxillary alveolus: a systematic review. Oral Radiol 2018; 34: 113-26.

4. Morris CR, Marano PD, Swimley DC, Runco JG. Abnormalities noted on panoramic radiographs. Oral Surg Oral Med Oral Pathol 1969; 28: 772-82.

5. MacDonald-Jankowski DS. The detection of abnormalities in the jaws: a radiological survey. Br Dent J 1991: 170: 215-8.

6. MacDonald D. Oral and maxillofacial radiology: a diagnostic approach. 2nd ed. Hoboken, NJ: Wiley-Blackwell; 2020.

7. Mallya S, Lam E. White and Pharoah's oral radiology: principles and interpretation. 8th ed. St. Louis, MO: Elsevier; 2019.

8. MacDonald-Jankowski DS, Orpe EC. Some current legal issues that may affect oral and maxillofacial radiology. Part 2: digital monitors and cone-beam computed tomography. J Can Dent Assoc 2007; 73: 507-11.
9. Alberta Government. Regional economic indicators. Peace Country region [Internet]. Edmonton: Treasury Board and Enterprise; 2011 [updated 2012 Mar 1; cited 2019 Oct 4]. Available from: https://open.alberta.ca/publications/1718-2840.

10. Statistics Canada [Internet]. Ottawa: Population and dwelling counts, for Canada, provinces and territories, 2011 and 2006 censuses [modified 2019 Jun 3; cited 2019 Oct 4]. Available from: https://www12.statcan.gc.ca/census-recensement/ 2011/dp-pd/hlt-fst/pd-pl/Table-Tableau.cfm?LANG=Eng\&T= $101 \& \mathrm{~S}=50 \& \mathrm{O}=\mathrm{A}$.

11. Kratz RJ, Nguyen CT, Walton JN, MacDonald D. Dental students' interpretations of digital panoramic radiographs on completely edentate patients. J Dent Educ 2018; 82: 313-21.

12. Kratz RJ, Walton JN, MacEntee MI, Nguyen CT, MacDonald D. Panoramic radiographs made before complete removable dental prostheses fabrication: a retrospective study of clinical significance. J Prosthet Dent 2017; 118: 26-30.

13. Lowry R. For a $2 \times 2$ contingency table [Internet]. Poughkeepsie (NY): VassarStats: website for statistical computation. C19882020 [cited 2019 Oct 4]. Available from: http://vassarstats.net/ tab2 2 .html.

14. McDonnell D. Dense bone island. A review of 107 patients. Oral Surg Oral Med Oral Pathol 1993; 76: 124-8.

15. MacDonald-Jankowski DS. Idiopathic osteosclerosis in the jaws of Britons and of the Hong Kong Chinese: radiology and systematic review. Dentomaxillofac Radiol 1999; 28: 357-63.

16. Takahashi A, Sugawara C, Kudoh T, Ohe G, Takamaru N, Tamatani $\mathrm{T}$, et al. Prevalence and imaging characteristics of palatine tonsilloliths evaluated on 2244 pairs of panoramic radiographs and CT images. Clin Oral Investig 2017; 21: 85-91.

17. Lim HK, Kim SM, Kim MJ, Lee JH. Clinical, statistical and chemical study of sialolithiasis. J Korean Assoc Oral Maxillofac Surg 2012; 38: 44-9.

18. MacDonald-Jankowski DS. Mucosal antral cysts observed within a London inner-city population. Clin Radiol 1994; 49: 195-8.

19. MacDonald-Jankowski DS. Mucosal antral cysts in a Chinese population. Dentomaxillofac Radiol 1993; 22: 208-10.

20. Horner K, MacDonald D. Conventional radiography in TMJ imaging. In: Rozylo-Kalinowska I, Orhan K. Imaging of the temporomandibular joint. Cham: Springer; 2019. p. 79-90.

21. MacDonald D, Horner K. Conventional radiographic findings of TMJ disorders. In Rozylo-Kalinowska I, Orhan K. Imaging of the Temporomandibular Joint. Cham: Springer; 2019. p. 91114.

22. Health Canada. Report on the findings of the oral health component of the Canadian health measures survey 2007-2009. Ottawa: Publications Health Canada; 2010 [cited Feb 7, 2020]. https://www.caphd.ca/sites/default/files/CHMS-E-tech.pdf.

23. Chu FC, Li TK, Lui VK, Newsome PR, Chow RL, Cheung LK. Prevalence of impacted teeth and associated pathologies - a radiographic study of the Hong Kong Chinese population. Hong Kong Med J 2003; 9: 158-63.

24. Canadian Dental Association. The state of oral health in Canada [Internet]. Ottawa: Canadian Dental Association; 2017 [cited 2019 Oct 4]. Available from: https://www.cda-adc.ca/stateoforalhealth/_files/TheStateofOralHealthinCanada.pdf.

25. Northern Alberta Development Council. Recommendations on 
health care in northern Alberta [Internet]. Alberta: Northern Alberta Development Council; 2007 [cited 2019 Oct 4]. Available from: http://www.nadc.gov.ab.ca/Docs/Health-Recommendations.pdf.

26. Hackbarth D. Report 76-4. Hydrogeology of the Grande Prairie area, Alberta [Internet]. Alberta: Alberta Research Council; 1977 [cited 2019 Oct 4]. Available from: https://ags.aer.ca/document/ESR/ESR_1976_04.pdf.

27. MacDonald D. Lesions of the jaws presenting as radiolucencies on cone-beam CT. Clin Radiol 2016; 71: 972-85.

28. Assaf AT, Solaty M, Zrnc TA, Fuhrmann AW, Scheuer H, Heiland M, et al. Prevalence of Stafne's bone cavity - retrospective analysis of 14,005 panoramic views. In Vivo 2014; 28: 1159-64.

29. Sisman Y, Miloglu O, Sekerci AE, Yilmaz AB, Demirtas O, Tokmak TT. Radiographic evaluation on prevalence of Stafne bone defect: a study from two centres in Turkey. Dentomaxillofac Radiol 2012; 41: 152-8.

30. Correll RW, Jensen JL, Rhyne RR. Lingual cortical mandibular defects: a radiographic incidence study. Oral Surg Oral Med Oral Pathol 1980; 50: 287-91.

31. Aoun G, Nasseh I, Diab HA, Bacho R. Palatine tonsilloliths: a retrospective study on 500 digital panoramic radiographs. J
Contemp Dent Pract 2018; 19: 1284-7.

32. Sigismund PE, Zenk J, Koch M, Schapher M, Rudes M, Iro H. Nearly 3,000 salivary stones: some clinical and epidemiologic aspects. Laryngoscope 2015; 125: 1879-82.

33. Kim SY, Kim HJ, Lim H, Lim MS, Kim M, Park IS, et al. Association between cholelithiasis and sialolithiasis: two longitudinal follow-up studies. Medicine (Baltimore) 2019; 98: e16153.

34. Vallo J, Suominen-Taipale L, Huumonen S, Soikkonen K, Norblad A. Prevalence of mucosal abnormalities of the maxillary sinus and their relationship to dental disease in panoramic radiography: results from the Health 2000 Health Examination Survey. Oral Surg Oral Med Oral Pathol Oral Radiol Endod 2010; 109: e80-7.

35. Larheim TA, Hol C, Ottersen MK, Mork-Knutsen BB, Arvidsson LZ. The role of imaging in the diagnosis of temporomandibular joint pathology. Oral Maxillofac Surg Clin North Am 2018; 30: 239-49.

36. MacDonald D, Chan A, Harris A, Vertinsky T, Farman AG, Scarfe WC. Diagnosis and management of calcified carotid artery atheroma: dental perspectives. Oral Surg Oral Med Oral Pathol Oral Radiol 2012; 114: 533-47. 